\title{
Localized versus delocalized states: Photoluminescence from electrochemically synthesized ZnO nanowires
}

\author{
T. Voss, ${ }^{1, a)}$ C. Bekeny, ${ }^{1}$ J. Gutowski, ${ }^{1}$ R. Tena-Zaera, ${ }^{2, b)}$ J. Elias, ${ }^{2}$ C. Lévy-Clément, ${ }^{2}$ \\ I. Mora-Seró, ${ }^{3}$ and J. Bisquert ${ }^{3}$ \\ ${ }_{1}^{1}$ Institute of Solid State Physics, University of Bremen, P.O. Box 330 440, D-28334 Bremen, Germany \\ ${ }^{2}$ Institut de Chimie et Matériaux de Paris-Est (ICMPE), CNRS, UMR 7182, Bât. F, 2-8 rue Henri Dunant, \\ 94320 Thiais, France \\ ${ }^{3}$ Departament de Física, Universitat Jaume I, Av. de Vicent Sos Baynat s/n, 12071 Castelló de la Plana, \\ Spain
}

(Received 26 May 2009; accepted 22 July 2009; published online 3 September 2009)

\begin{abstract}
We analyze the near-band-edge photoluminescence of electrochemically deposited $\mathrm{ZnO}$ nanowires and directly correlate the photoluminescence properties with the carrier concentration in the nanowires as determined from electrochemical impedance spectroscopy. We find a donor density of $8 \times 10^{19} \mathrm{~cm}^{-3}$ in the as-deposited nanowires and show that the near-band-edge emission results from band-to-band recombination processes (delocalized states). A photoluminescence band centered at $3.328 \mathrm{eV}$ scales with the diameter of the nanowires and is assigned to recombination processes involving surface states. We show that annealing at $500{ }^{\circ} \mathrm{C}$ in air reduces the donor density in the nanowires by more than one order of magnitude, leading to sharp excitonic transitions in the electrochemically deposited nanowires. (C) 2009 American Institute of Physics.

[doi:10.1063/1.3211294]
\end{abstract}

\section{INTRODUCTION}

$\mathrm{ZnO}$ nanowires have been studied in great detail during the past years because they offer interesting properties that might be used for nanoscale light emitters, subwavelength waveguides, electrical nanosensors with increased sensitivity, or solar-cell applications. ${ }^{1-3}$ In general, the nanowires can be characterized as $\mathrm{ZnO}$ single crystals with a large aspect ratio, i.e., their length in $c$-axis direction is significantly exceeding all other lateral dimensions, with the latter ones being in the 10 to some hundred nanometer range. This implies that nanowires have a significantly increased surfaceto-volume ratio when compared to standard bulk crystals, resulting in considerably strong surface effects ${ }^{4}$ in nanowirebased optoelectronic devices.

For many of such devices, a large scale, low-cost fabrication of high-quality and single crystalline $\mathrm{ZnO}$ nanowires with a high degree of $c$-axis orientation is desirable. Such demands are met by low-temperature chemical synthesis methods. The electrochemical deposition (ECD) of $\mathrm{ZnO}$ at temperatures below $100{ }^{\circ} \mathrm{C}$, based on the reduction of dissolved molecular oxygen in aqueous solutions containing $\mathrm{Zn}^{2+}$ ions, ${ }^{5}$ has recently shown promising results in obtaining arrays of single crystalline $\mathrm{ZnO}$ nanowires with tailored dimensions. ${ }^{6-9}$ Because of the aqueous environment, the limited purity of the involved chemicals and the low growth temperatures (especially when compared to typical growth temperatures used for vapor-phase epitaxial methods) is not a priori clear how the nanowire material compares to that of nanowires synthesized by, e.g., high-temperature vapor-

\footnotetext{
${ }^{\text {a)}}$ Electronic mail: voss@ifp.uni-bremen.de.

${ }^{b)}$ Present address: New Materials Department, CIDETEC, Center for Electrochemical Technologies, Parque Tecnológico de San Sebastián, Paseo Miramón 196, Donostia-San Sebastián 20009, Spain.
}

liquid-solid processes. ${ }^{10}$ However, a detailed knowledge of the microscopic processes governing the optical and electronic properties of chemically synthesized nanowires is mandatory to understand, tailor, and optimize any optoelectronic device based on such nanowires.

In this paper, we report on the identification of the processes that contribute to the near-band-edge emission (NBE) in electrochemically synthesized nanowires. We show that the relatively high donor concentration in the as-deposited nanowires leads to emission from delocalized donor-band states as opposed to localized bound-exciton states where the latter ones usually dominate the optical properties of $\mathrm{ZnO}$ with moderate donor densities at liquid-helium temperature. We show that annealing of the nanowires at moderate conditions reduces the donor density by about an order of magnitude and results in the observation of sharp emission bands associated with bound-exciton transitions after the annealing process.

\section{EXPERIMENTAL}

The $\mathrm{ZnO}$ nanowire arrays were electrochemically deposited on conducting $(10 \Omega / \mathrm{sq})$ glass $/ \mathrm{SnO}_{2}: F$ from Solaronix, covered by a $\mathrm{ZnO}$ nanocrystalline buffer layer. This layer was fabricated by two different techniques: Spray pyrolysis and electrodeposition. ${ }^{6}$ The sizes of nanocrystals determine the diameters of the nanowires because they act as nucleation centers for the nanowire growth. Details about the electrodeposition of the $\mathrm{ZnO}$ nanowire arrays can be found in Refs. 6 and 7. Briefly, the electrodeposition was performed at $80{ }^{\circ} \mathrm{C}$ under constant potential $(-1 \mathrm{~V}$ versus saturated calomel electrode). The electrolyte was an aqueous solution of $\mathrm{ZnCl}_{2}$ (Flucka purity $>98 \%$ ) and $\mathrm{KCl}$ (Flucka purity $>99.5 \%$ ) saturated with bubbling oxygen $10 \mathrm{~min}$ before and 
during the experiment. The zinc chloride and potassium chloride concentrations $\left(\left[\mathrm{ZnCl}_{2}\right]\right.$ and $\left.[\mathrm{KCl}]\right)$ were 5 $\times 10^{-4} M$ and $0.1 M$, respectively, for all samples.

For optical characterization, a microphotoluminescence (PL) setup was used with a frequency-doubled Ti-sapphire laser with a repetition rate of $82 \mathrm{MHz}$ at $3.54 \mathrm{eV}$ as excitation source (excitation fluence $\sim 10 \mu \mathrm{J} / \mathrm{cm}^{-2}$ ). The sample was placed in a temperature-variable cryostat cooled with liquid helium. The laser light was focused on the sample through a microscope objective (spot diameter on sample: $2 \mu \mathrm{m})$. The PL from the nanowires was collected via the same objective, spectrally resolved by a spectrometer (resolution $0.06 \mathrm{~nm}$ ) and detected by a liquid-nitrogen-cooled charge coupled device camera.

To determine the donor density in the nanowires, electrochemical impedance spectroscopy (EIS) measurements were performed in a three-electrode cell using a Pt wire as counter electrode and a standard $\mathrm{Ag} / \mathrm{AgCl}$ wire $(3 \mathrm{M} \mathrm{KCl})$ as the reference electrode. A carbonate propylene electrolyte $\left(0.1 M \mathrm{LiClO}_{4}\right)$ was used to avoid $\mathrm{ZnO}$ nanowire decomposition. The EIS data were obtained using a potentiostat (Autolab PGSTAT-30) equipped with a frequency-response analyzer. Each measurement was done by applying a $20 \mathrm{mV}$ ac sinusoidal signal with the frequency ranging from $500 \mathrm{kHz}$ down to $5 \mathrm{mHz}$ superimposed to the applied constant bias. The donor density determined by the EIS is assumed to be equal to the density of the activated donors at room temperature where the EIS measurements are performed. For a detailed description of the EIS technique in which we carefully account for the influence of the buffer layer, see Ref. 11.

For the annealing process, $\mathrm{ZnO}$ samples were placed in a specially designed sample holder in an oven (Nabertherm, model L3/11/B170) and heated up to the required temperature. The sample holder was equipped with a gas inlet enabling the annealing process to be carried out in a specific atmosphere. The heating rate of the sample was $6{ }^{\circ} \mathrm{C} / \mathrm{min}$. The sample was annealed at $500{ }^{\circ} \mathrm{C}$ for $1 \mathrm{~h}$, taken out of the oven and being allowed to cool down naturally to room temperature.

\section{RESULTS}

In Fig. 1, the PL spectra of the NBE of an as-grown $\mathrm{ZnO}$ nanowire array at temperatures between $T=4$ and $290 \mathrm{~K}$ are depicted. For this nanowire array, the density of activated donors at room temperature determined from EIS is $N_{D}=8$ $\times 10^{19} \mathrm{~cm}^{-3}$. The spectrum at $T=4 \mathrm{~K}$ shows two main broad emission bands: One centered at $E=3.328 \mathrm{eV}$ with a linewidth of $\Delta E=23 \mathrm{meV}$ (labeled $D$ ), and one at $E$ $=3.366 \mathrm{eV}$ with a linewidth of $\Delta E=18 \mathrm{meV}$ (labeled DB). Both emission bands have asymmetric shapes with a smooth decrease toward lower energies. With increasing temperature, both the DB and $D$ emission bands broaden and merge above $T=60 \mathrm{~K}$ while the whole NBE shifts toward lower energies, as expected for the NBE due to the temperature dependence of the band gap. Additional, significantly weaker emission bands at $E \sim 3.2$ and $E \sim 3.1 \mathrm{eV}$ can be observed at room temperature, which are most probably related to longitudinal optical phonon replica of the broad $D$ and DB bands.

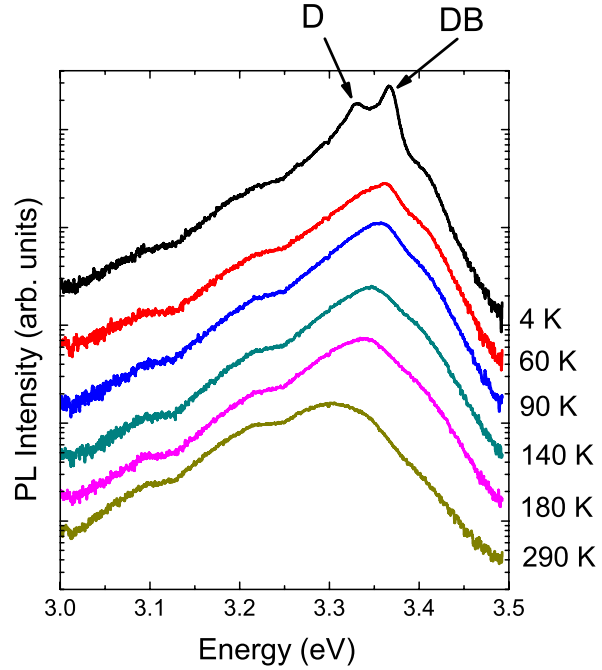

FIG. 1. (Color online) Near-band-edge PL spectrum of an as-deposited ZnO nanowire array measured at temperatures between 4 and $290 \mathrm{~K}$.

Figure 2 shows SEM micrographs of arrays of the $\mathrm{ZnO}$ nanowire samples with the (a) smallest and (b) largest diameters analyzed in this work. The variation in the diameter in the electrodeposition process was achieved by changing the grain size of the nanocrystals which act as seed crystals. ${ }^{6}$ This allows for the tailoring of the nanowire diameter while keeping all other parameters constant for the growth process of the nanowires. The mean value of the nanowire diameter was estimated from a statistical evaluation of the SEM micrographs. ${ }^{9}$

Figure 3(a) compares the low-temperature NBE spectra between 3.30 and $3.34 \mathrm{eV}$ for three arrays of as-deposited $\mathrm{ZnO}$ nanowires with different mean diameters. The spectra are normalized to the maximum of the DB peak. The error bar on each spectrum indicates the variation of the intensity at various excitation positions on the ensemble, obtained due to dispersion in nanowire diameter in each sample. ${ }^{9}$ The average diameter $d$ of the nanowires on each array is given in the figure. For smaller diameters, we observe an increasing contribution of the $D$ emission band to the PL spectrum. Figure 3(b) reveals an almost linear dependence of the emission intensity ratio $\left(I_{D} / I_{\mathrm{DB}}\right)$ on the inverse diameter of the nanowire $(1 / d)$.

Postgrowth annealing treatment of low-temperature grown nanowires under appropriate conditions can enormously improve their optical quality. ${ }^{12}$ In Fig. 4 , the PL spectra of $\mathrm{ZnO}$ nanowires annealed at $T=500{ }^{\circ} \mathrm{C}$ for $60 \mathrm{~min}$ in air are depicted. The spectra are measured at temperatures

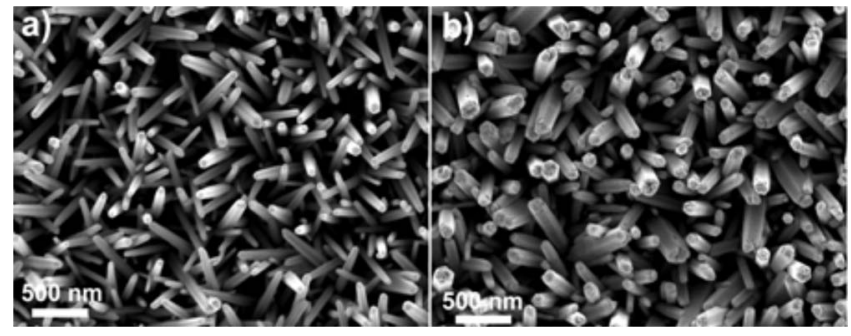

FIG. 2. SEM micrographs of two arrays of $\mathrm{ZnO}$ nanowires with the (a) smallest and the (b) largest average diameter investigated. 


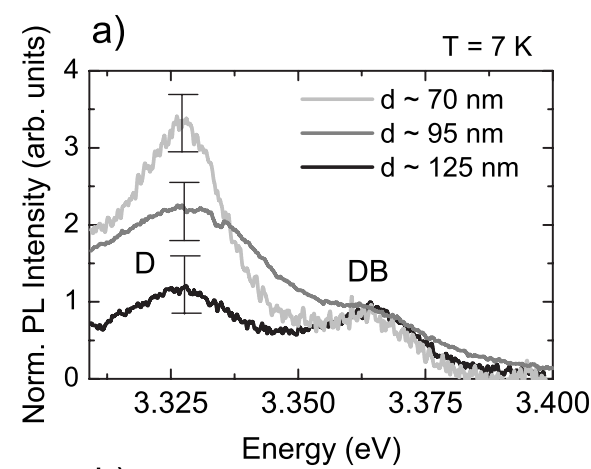

b)

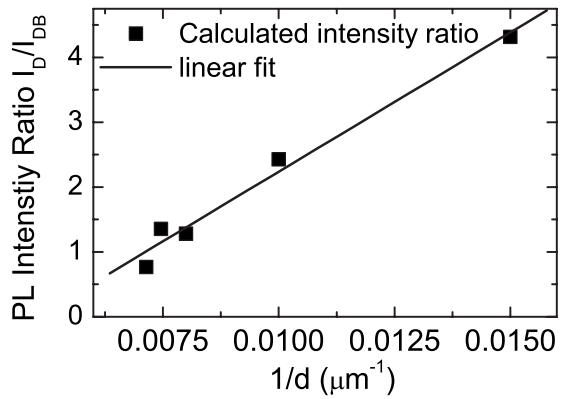

FIG. 3. (a): Low temperature PL spectra of as-deposited $\mathrm{ZnO}$ nanowire arrays with different average diameters. The error bar on each spectrum indicates the variation in the intensity at various excitation positions on the ensemble. (b): Dependence of the intensity ratio of the $D$ and DB bands $\left(I_{D} / I_{\mathrm{DB}}\right)$ on the inverse of the nanowire diameter $1 / d$.

between $T=7$ and $140 \mathrm{~K}$. After the annealing process, distinct and relatively sharp emission lines are detected at $T$ $=7 \mathrm{~K}$, contrasting the spectrum measured for the asdeposited $\mathrm{ZnO}$ nanowire array (compared to Fig. 1). From its temperature dependence and energy position, the emission shoulder at $3.376 \mathrm{eV}$ is assigned to free-exciton transitions $(\mathrm{FX}){ }^{2,13}$ The main emission band $D^{0} X$ at $3.361 \mathrm{eV}$ (linewidth $\Delta E=2 \mathrm{meV}$ ) is attributed to (localized) donor-bound exciton transitions. ${ }^{2,13}$ An additional emission band $(B)$ is observed at $3.318 \mathrm{eV}$, its origin will be discussed in Sec. IV. The SD emission band at $3.331 \mathrm{eV}$ results from recombination processes involving excitons bound to structural

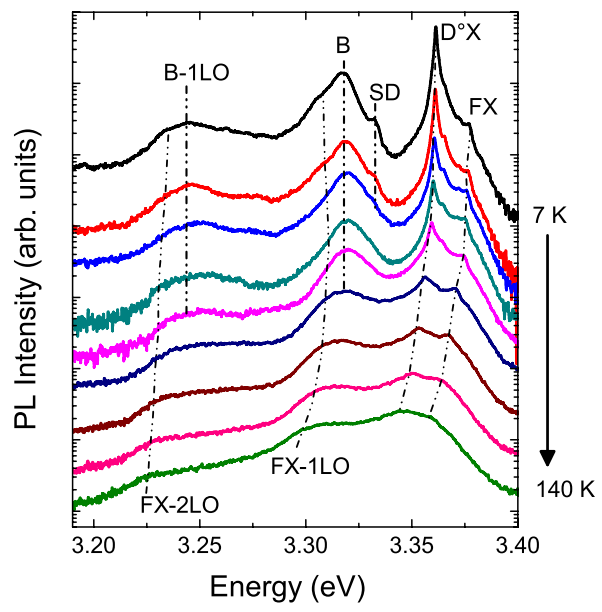

FIG. 4. (Color online) (a): Near-band-edge PL spectra of a $\mathrm{ZnO}$ nanowire array annealed at $T=500{ }^{\circ} \mathrm{C}$ for $60 \mathrm{~min}$ in air measured between $T=7 \mathrm{~K}$ and $T=140 \mathrm{~K}$. The dotted lines are guides to the eye. defects. ${ }^{13}$ The 1-LO-phonon replica of the FX (FX-1LO) and the $B$ transitions $(B-1 \mathrm{LO})$ are visible at an energy separation of $72 \mathrm{meV}$ to the lower energy side of their respective zerophonon lines. ${ }^{2,10}$ All bands shift to lower energies as the temperature increases due to the reduction of the band gap. The intensities of the $D^{0} X$ and $B$ bands decrease while those of the FX and FX-1LO bands increase. The SD band is hardly visible above $T=40 \mathrm{~K}$. Above $T=140 \mathrm{~K}$, the FX$1 \mathrm{LO}$ emission band dominates the spectrum.

\section{DISCUSSION}

The results presented in the previous section demonstrate that, without postgrowth annealing treatment, the donor concentration in electrochemically synthesized $\mathrm{ZnO}$ nanowires is usually relatively high, especially when compared to $\mathrm{ZnO}$ nanowires synthesized by high-temperature vapor-transport techniques for which carrier densities on the order of $10^{17} \mathrm{~cm}^{-3}$ have been reported. ${ }^{14}$ For our samples, the value $N_{D}=8 \times 10^{19} \mathrm{~cm}^{-3}$ determined by EIS readily explains the broad linewidth of the DB emission in the PL spectrum of Fig. 1. In the presence of such high donor densities, a band of donor states is formed due to the overlap of the wave functions of neighboring donors. ${ }^{12}$ Recombination processes take place between this band and the valence band (band-to-band transitions) as opposed to transitions involving localized (bound-)exciton states (in nominally undoped $\mathrm{ZnO}$ known as $D^{0} X$ transitions) which usually dominate the optical spectra of $\mathrm{ZnO}$ crystals at low temperatures. ${ }^{2,12,13}$

The Mott criterion ${ }^{15}$ states that there exists a critical donor density $N_{C}$ beyond which the wave functions of the donors are so close to each other that they overlap and form a band of donor states. $N_{C}$ is calculated from the equation

$$
a_{B} \cdot N_{C}^{1 / 3}=0.24 \text {. }
$$

$a_{B}$ is the exciton Bohr radius $\left(a_{B}^{\mathrm{ZnO}}=1.8 \mathrm{~nm}\right.$ for the $A$ and $B$ excitons). ${ }^{16}$ Therefore, we find a critical donor density of $N_{C}=2.4 \times 10^{18} \mathrm{~cm}^{-3}$. For the electrochemically deposited nanowires, $N_{D}>N_{C}$, which supports the above given interpretation that the PL emission originates from delocalized donor-band states.

As can be seen in Fig. 3, the relative intensity of the $D$ band (normalized to the DB intensity $I_{\mathrm{DB}}$ ) scales with the inverse of the nanowire diameter according to $I_{D} / I_{\mathrm{DB}} \propto 1 / d$; the relative intensity increases by a factor of 3.5 when the diameter of the nanowire is reduced from 125 to $70 \mathrm{~nm}$. We therefore assign the $D$ peak to recombination processes involving surface states which increasingly contribute to the overall PL emission for smaller wire diameters as the surface-to-volume ratio increases.

The quality-improving influence of thermal annealing is demonstrated in Fig. 4 by the recovery of sharp excitonrelated transition lines. The donor density determined for the annealed samples is $4 \times 10^{18} \mathrm{~cm}^{-3}$ at $T=300 \mathrm{~K}$, a factor of 10 less than $N_{D}$ in the as-deposited nanowires. $N_{D}$ determined for the annealed samples is therefore of the same order as the above calculated critical donor density $N_{C}$ thus explaining the observation of exciton-related recombination process. The reduction in $N_{D}$ during the annealing process is 
due to the out-diffusion of the residual shallow donors, most probably Zn interstitials. ${ }^{17,18}$ Furthermore, the absence of the $D$ emission band might be attributed to surface reconstruction in the annealed nanowires.

The $B$ emission band observed at $3.318 \mathrm{eV}$ shows a similar temperature dependence as the main $D^{0} X$ band. We carried out intensity dependent measurements (not shown here) in which both the $B$ and the $D^{0} X$ bands showed a linear dependence on the excitation fluence. This suggests that both emission bands have a similar origin ${ }^{19}$ (i.e., donor-bound excitons) and both bands are visible as long as the excitonic transitions determine the emission spectrum. Therefore, we conclude that the $B$ emission band results from a donorbound exciton transitions with the donor involved being a deep one, most probably a Cl-related complex state. More investigations are in progress to identify its nature.

All in all, the near-band-edge PL from electrochemically deposited $\mathrm{ZnO}$ nanowires can be completely understood by considering the donor densities determined by EIS. The presence of a band of donor states and, thus, band-to-band transitions as opposed to transitions involving localized boundexciton states is a crucial point to consider when discussing the performance of nanowire-based solar cells, light-emitting diodes, and other optoelectronic devices. In particular, our results demonstrate that for as-grown ECD nanowires, excitonic effects should play no role for the PL emission processes due to the high concentration of activated donors. Furthermore, distinguishable and localized excitonic transitions, similar to those observed in $\mathrm{ZnO}$ nanowires and crystals grown by high-temperature vapor-transport processes, are clearly visible in the PL spectra after annealing the samples in air. This can be attributed to a significant reduction of the donor density by the still relatively mild annealing process.

\section{CONCLUSIONS}

In summary, we have shown that the broad NBE observed at $3.366 \mathrm{eV}$ in electrochemically deposited $\mathrm{ZnO}$ nanowires results from band-to-band transitions. We have explained the absence of excitonic states and the formation of a band of donor states by considering the Mott criterion. Donor densities higher than $8 \times 10^{19} \mathrm{~cm}^{-3}$ have been determined for the as-deposited $\mathrm{ZnO}$ nanowires at room temperature. Furthermore, a second emission band $D$ scales with the diameter of the nanowires and is attributed to recombination processes involving surface states. Annealing the nanowires at $500{ }^{\circ} \mathrm{C}$ for $60 \mathrm{~min}$ in air results in a reduction of the donor density by about one order of magnitude and the observation of sharp emission bands associated with bound-exciton transitions. The results show a very good correlation of the electrical and optical properties of the electrochemically deposited $\mathrm{ZnO}$ nanowires.

\section{ACKNOWLEDGMENTS}

The authors wish to thank Ilja Rückmann for support and fruitful discussions. This work has been supported by the BFK University of Bremen, the German Research Foundation (Grant No. DFG Vo1265/4-1/2), the Ministerio de Educacion y Ciencia of Spain under Grant No. HOPE CSD200700007 (Consolider-Ingenio 2010), and the Generalitat Valenciana under the Grant No. GV06/347.

${ }^{1}$ C. Klingshirn, ChemPhysChem 8, 782 (2007).

${ }^{2}$ Ü. Özgür, Y. I. Alivov, C. Liu, A. Teke, M. A. Reshchikov, S. Dogan, V. Avrutin, S. J. Cho, and H. Morkoc, J. Appl. Phys. 98, 041301 (2005).

${ }^{3}$ C. Levy-Clement, R. Tena-Zaera, M. A. Ryan, A. Katty, and G. Hodes, Adv. Mater. 17, 1512 (2005).

${ }^{4}$ L. Wischmeier, T. Voss, I. Rückmann, J. Gutowski, A. C. Mofor, A. Bakin, and A. Waag, Phys. Rev. B 74, 195333 (2006).

${ }^{5}$ S. Peulon and D. Lincot, J. Electrochem. Soc. 145, 864 (1998).

${ }^{6}$ J. Elias, R. Tena-Zaera, and C. Levy-Clement, Thin Solid Films 515, 8553 (2007)

${ }^{7}$ R. Tena-Zaera, J. Elias, G. Wang, and C. Levy-Clement, J. Phys. Chem. C 111, 16706 (2007).

${ }^{8}$ J. Elias, R. Tena-Zaera, and C. Levy-Clement, J. Electroanal. Chem. 621, 171 (2008).

${ }^{9}$ J. Elias, R. Tena-Zaera, and C. Levy-Clement, J. Phys. Chem. C 112, 5736 (2008).

${ }^{10}$ T. Voss, C. Bekeny, L. Wischmeier, H. Gafsi, S. Börner, W. Schade, A. C. Mofor, A. Bakin, and A. Waag, Appl. Phys. Lett. 89, 182107 (2006).

${ }^{11}$ I. Mora-Sero, F. Fabregat-Santiago, B. Denier, J. Bisquert, R. Tena-Zaera, J. Elias, and C. Levy-Clement, Appl. Phys. Lett. 89, 203117 (2006).

${ }^{12}$ C. Bekeny, T. Voss, H. Gafsi, J. Gutowski, B. Postels, M. Kreye, and A. Waag, J. Appl. Phys. 100, 104317 (2006).

${ }^{13}$ B. K. Meyer, H. Alves, D. M. Hofmann, W. Kriegseis, D. Forster, F. Bertram, J. Christen, A. Hoffmann, M. Straßburg, M. Dworzak, U. Haboeck, and A. V. Rodina, Phys. Status Solidi B 241, 231 (2004).

${ }^{14}$ P.-C. Chang, C.-J. Chien, D. Stichtenoth, C. Ronning, and J. G. Lu, Appl. Phys. Lett. 90, 113101 (2007).

${ }^{15}$ N. F. Mott, Metal-Insulator Transition (Taylor and Francis, London, 1974).

${ }^{16}$ Landolt-Börnstein, Group III: Condensed Matter, Subvolume B: II-VI and I-VIII Compounds, Semimagnetic Compounds, edited by O. Madelung, U. Rössler, and M. Schulz (Springer, Heidelberg, 1999) Vol. 41.

${ }^{17}$ D. C. Look, G. C. Farlow, P. Reunchan, S. Limpijumnong, S. B. Zhang, and K. Nordlund, Phys. Rev. Lett. 95, 225502 (2005).

${ }^{18}$ P. Erhart and K. Albe, Appl. Phys. Lett. 88, 201918 (2006).

${ }^{19}$ J. Gutowski, N. Presser, and I. Broser, Phys. Rev. B 38, 9746 (1988). 